\title{
Comparison between Simulation and Experimentally Observed Interactions between Two Magnetic Beads in a Fluidic System
}

\author{
Olayinka Oduwole, David Tim Grob, Steve Sheard. \\ Department of Engineering Science, University of Oxford, Parks Road, Oxford, OX1 3PJ, \\ United Kingdom.
}

\begin{abstract}
Continuous flow separation of magnetic particles within a microfluidic device could lead to improved performance of magnetic bead-based assays but the undesirable formation of bead clusters reduces its efficiency; this efficiency refers to the ability to separate bound magnetic beads from a mixture of particles. Such agglomerates are formed due to magnetic binding forces while hydrodynamic interactions strongly influence the particles' movement. This article presents a model for interactions between a pair of equal sized superparamagnetic beads suspended in water within a uniform magnetic field. To the best of our knowledge, we present for the first time a comparison between simulated trajectories and the beads' movement captured on video; the beads were suspended in a stationary fluid placed within a uniform magnetic field. In conclusion, the model is a good approximation for beads interacting with their nearest neighbours and is able to predict the trajectory pattern of these particles in a magnetic bead-based assay. Predicting the magnetically induced interaction of nearby beads will help in determining the density of beads in an assay and in avoiding agglomeration over a fixed time duration.
\end{abstract}

Keywords: Super-paramagnetic bead; Magnetic Separation; Magnetic Interaction; Hydrodynamic Interaction; Microfluidics; Magnetic Field.

\section{Introduction}

The concept of magnetic particle separation has been utilized in various diagnostic applications such as immuno-magnetic assays, where a target bio-molecule (cells, proteins, antibodies, bacteria, DNA, RNA etc.) is bound to a magnetic particle; the targeted biomolecule is then isolated from a mixture using the magnetic properties of the complex. This is typically achieved using a test-tube arrangement but now there is growing interest in using microfluidics for this application due to (i) commercially available magnetic beads coated with specific bio-molecules, (ii) the ability to handle low sample volume, (iii) the potential for faster processing time and (iv) recent advances in microfluidic technology [1, 2]. Furthermore, the small size of these microfluidic devices allows for improved integration with other components such as pumps, valves and switches to form a micro total analysis system [1, 2, $3]$. These microfluidic applications have been utilized in cell sorting $[4,5]$, cell capture and manipulation $[2,3,6,7]$, purification and separation $[3,6,8]$ techniques for various biological and biomedical purposes. 
Formation of bead agglomerates due to particle-particle interactions have been previously observed during magnetic separation [9]. Agglomeration causes surface deposits, obstructs the channel and reduces the separation efficiency of these devices. In order to improve the design of these devices, it is important to determine how magnetic particles move and interact with one another when suspended in fluid within a magnetic field.

In this study, we present a two dimensional numerical model for a pair of equal sized super-paramagnetic beads suspended in water within a uniform magnetic field moving towards each other when responding to magnetic and hydrodynamic interactions. Each bead leads to a disturbance in the magnetization and local field of the other bead, giving rise to magnetic interactions; this has been previously modelled analytically by Mikkelsen et al., Banerjee et al. [10, 11]. Hydrodynamic interaction describes the impact of a bead moving in fluid on its neighbouring bead(s); we applied the method of reflection $[12,13]$ and included the lubrication approximation $[14,15]$ in the simulation to model this interaction.

Following Banerjee et al. [11] we model the trajectory pattern of the bead pair within the uniform magnetic field but go further by validating these results with data obtained from captured video. We estimated the agglomeration time as a function of the separation distance for a magnetic bead-based assay. This pair interaction model is particularly relevant to magnetic separation devices that utilise a low density of beads. Previous studies [16, 17] have modelled particle interactions for devices with higher bead density.

\section{Theoretical Model}

The formation of bead clusters within a magnetic separation device occurs due to magnetic binding forces and hydrodynamic interactions. In this study, we consider a pair of superparamagnetic beads suspended in a viscous fluid within a uniform magnetic field.

Using Newton's second law of motion and the assumption that the beads reach their terminal velocity quickly, the equation of motion can be written as

$\sum \mathbf{F}=0$

where $\mathbf{F}$ is the Force in $\mathbf{N}$. Here, the bold letter indicates a vector.

The forces that will be considered in this study are gravity, buoyancy, magnetic force, drag and hydrodynamic interactions. All forces will be resolved within the x-y plane assuming the pair of magnetic beads move in this plane only.

\subsection{Magnetic Force}

This is the force that acts on the magnetic bead due to the presence of an external magnetic field. A gradient in the magnetic field leads to an in-homogeneity which gives rise to the motion of the magnetic bead. For the case of a uniform magnetic field, this translational force is zero. 
If a second super-paramagnetic bead is introduced within the uniform magnetic field, its presence leads to an induced magnetization and a perturbed external field; this inhomogeneity in the uniform magnetic field leads to an interaction force which causes the beads to move with respect to one another [10].

The magnetic force interaction, $\mathbf{F}_{\mathbf{m}}$ is given by

$$
\mathbf{F}_{\mathbf{m}}=\int \mu_{o}\left(\left(\mathbf{M}_{1}+\mathbf{d} \mathbf{M}_{1}\right) \cdot \boldsymbol{\nabla}\right)\left(\mathbf{H}_{\mathbf{e x t}}+\mathbf{d} \mathbf{H}_{2}\right) d V
$$

where $\mu_{o}$ is vacuum permeability, $\mathbf{M}_{\mathbf{1}}$ is the magnetization of the first bead in $\mathrm{A} / \mathrm{m}, \mathbf{d} \mathbf{M}_{\mathbf{1}}$ is the induced magnetization due to the presence of the second bead in $\mathrm{A} / \mathrm{m}, \mathbf{H}_{\text {ext }}$ is the external field in $\mathrm{A} / \mathrm{m}, \mathbf{d H}_{\mathbf{2}}$ is the perturbed external field due to the presence of the second bead in $\mathrm{A} / \mathrm{m}$ and $V$ is the volume of the bead in $\mathrm{m}^{3}$ (See Appendix for more information) [10].

\subsection{Drag Force}

This is the force that opposes the motion of a particle within a viscous fluid. In this study, the fluid is stationary and the Reynolds' number for a moving bead within this fluid is within $10^{-5}$ to $10^{-6}$; the Navier-Stokes' equation can be simplified by neglecting the inertia and convective terms while considering the viscous terms. The drag force, $\mathbf{F}_{\mathbf{d}}$ is given by

$\mathbf{F}_{\mathbf{d}}=-6 \pi \eta a(\mathbf{u}-\mathbf{v})$

where $a$ is the radius of the bead in $\mathrm{m}, \eta$ is the viscosity of the fluid in $\mathrm{kg} / \mathrm{ms}, \mathbf{u}$ is the velocity of the bead in $\mathrm{m} / \mathrm{s}$ and $\mathbf{v}$ is the velocity field created by the motion of the two beads in $\mathrm{m} / \mathrm{s}$. (see next section).

\subsection{Hydrodynamic Interaction}

This is an interaction that arises due to the fluid environment and the presence of the other bead within the fluid. It could be either long-range which occurs when the beads are separated by a gap-distance greater than their diameter or short-range when otherwise.

\subsubsection{Long-Range Hydrodynamic Interaction}

In general, when a single bead moves in a stationary fluid, the fluid in near contact with the bead is dragged along at its speed. This creates a disturbance in the fluid which can be described as a velocity field. This velocity field is assumed to be reflected towards the other bead leading to a change in its flow pattern and effective velocity.

The method of reflection is used to evaluate the resultant velocity field and drag force on the beads [12]. This method is linear and subdivides the velocity and pressure into a sum of terms that satisfy the Navier-Stokes' equation and the boundary conditions of the fluid at each step. The resultant velocity of the bead is then obtained as an infinite sum comprising its initial velocity and the successive reflected velocity terms due to the presence of the other bead [12, 13]. 
Assume $\mathbf{r}$ and $\mathbf{n}$ represent the vector along and normal to the line of centres of the magnetic beads respectively, the long-range hydrodynamic force interaction, $\mathbf{F}_{\mathbf{h}}$ on a single bead can be written as

$$
\begin{aligned}
& \mathbf{F}_{\mathbf{h}}=-6 \pi \eta a_{1}\left(\frac{\left(\left(\mathbf{u}_{\mathbf{1}} \cdot \hat{\mathbf{r}}\right)-k_{p 1}\left(\mathbf{u}_{2} \cdot \hat{\mathbf{r}}\right)\right)}{\left(1-k_{p 1}{ }^{2}\right)} \hat{\mathbf{r}}+\frac{\left(\left(\mathbf{u}_{\mathbf{1}} \cdot \widehat{\mathbf{n}}\right)-k_{n 1}\left(\mathbf{u}_{2} \cdot \widehat{\mathbf{n}}\right)\right)}{\left(1-k_{n 1}{ }^{2}\right)} \widehat{\mathbf{n}}\right) \\
& k_{p 1}=\left(\frac{3}{2}\left(\frac{a_{2}}{d}\right)-\frac{1}{2}\left(\frac{a_{2}}{d}\right)^{3}\right) \\
& k_{n 1}=\left(\frac{3}{4}\left(\frac{a_{2}}{d}\right)+\frac{1}{4}\left(\frac{a_{2}}{d}\right)^{3}\right)
\end{aligned}
$$

where the subscripts 1 and 2 represent each of the beads respectively and $d$ is the centre-tocentre distance between the beads in $\mathrm{m}[12,13]$.

\subsubsection{Short-Range Hydrodynamic Interaction}

Lubrication effects come into play when two particles are nearly in contact and the squeezed fluid in between them sets up a high velocity gradient and pressure distribution that restrains them from coming into closer contact $[14,15]$. This occurs when the beads are separated by a gap-distance comparable to their physical dimensions.

If two beads move in a viscous fluid with a relative velocity, $\mathbf{u}_{\mathbf{r}}$, towards each other, they experience a lubrication force given by

$$
\begin{aligned}
& \mathbf{F}_{\text {lub }}=-\frac{3 \pi \eta a_{h} a_{g}\left(\mathbf{u}_{\mathbf{r}} \cdot \hat{\mathbf{r}}\right) \hat{\mathbf{r}}}{d_{o}} \\
& d_{o}=|d|-\left(a_{1}+a_{2}\right)
\end{aligned}
$$

where $d_{o}$ is the gap-distance in $\mathrm{m}, a_{h}$ is the harmonic mean of the radii of both beads in $\mathrm{m}$ and $a_{g}$ is the geometric mean of the radii of both beads in $\mathrm{m}[14,15]$.

\subsection{Brownian Motion}

An observation of the motion of super-paramagnetic beads suspended in water reveals randomness in their movement; this random movement occurs due to the bombardment of the beads by the water molecules and is termed 'Brownian motion'. The mean squared displacement of a Brownian particle in two dimensions (2D) is related to the diffusion coefficient by

$<\Delta r^{2}>_{2 D}=4 D t_{s}$ 
$D=\frac{k T}{6 \pi \eta a}$

where $D$ is the diffusion coefficient in $\mathrm{m}^{2} / \mathrm{s}, k$ is the Boltzmann's constant, $T$ is the temperature in kelvin, $t_{s}$ is the sampling time in $\mathrm{s}, \Delta r^{2}$ is the mean squared displacement in $\mathrm{m}^{2}$ [18].

\subsection{Equation of Motion}

The resultant force acting on one of the beads when affected by the presence of a second bead is expressed as $\mathbf{F}_{\mathbf{r}}$. The velocity of the bead, $\mathbf{u}_{\mathbf{1}}$ can be resolved along and normal to the line of centres between the beads as

$$
\begin{aligned}
& \mathbf{u}_{\mathbf{1}}=A \hat{\mathbf{r}}+B \widehat{\mathbf{n}} \\
& A=\frac{C}{D} \\
& C=\frac{\left(\mathbf{F}_{\mathbf{r}} \cdot \hat{\mathbf{r}}\right)\left(1-k_{p 1}^{2}\right)}{6 \pi \eta a_{1}}+\mathbf{u}_{2} \cdot \hat{\mathbf{r}}\left(k_{p 1}+\frac{a_{n}}{2 d_{o}}\left(1-k_{p 1}^{2}\right)\right) \\
& D=\left(1+\frac{a_{n}}{2 d_{o}}\left(1-k_{p 1}^{2}\right)\right) \\
& B=\left(\frac{\left(\mathbf{F}_{\mathbf{r}} \cdot \widehat{\mathbf{n}}\right)\left(1-k_{n 1}^{2}\right)}{6 \pi \eta a_{1}}+k_{n 1}\left(\mathbf{u}_{2} \cdot \widehat{\mathbf{n}}\right)\right) \\
& a_{n}=\frac{a_{g} a_{h}}{2 a_{1} d_{o}}
\end{aligned}
$$

Using a finite-time-finite-difference scheme [19], the position of bead $1, \mathbf{r}_{1}$ is initially estimated as

$\mathbf{r}_{\mathbf{1}}^{*}(\mathrm{n}+1)=\mathbf{r}_{\mathbf{1}}(\mathrm{n})+\mathbf{u}_{\mathbf{1}}\left(\mathbf{r}_{\mathbf{1}}(\mathrm{n})\right) d t$

Using a predictor corrector method [19], the position vector is then determined as

$\mathbf{r}_{\mathbf{1}}(\mathrm{n}+1)=\mathbf{r}_{\mathbf{1}}(\mathrm{n})+\frac{1}{2}\left(\mathbf{u}_{\mathbf{1}}\left(\mathbf{r}_{\mathbf{1}}(\mathrm{n})\right)+\mathbf{u}_{\mathbf{1}}\left(\mathbf{r}_{\mathbf{1}}^{*}(\mathrm{n}+1)\right)\right) d t$ 
This process is repeated for the second bead. The numerical model is implemented in Matlab for a pair of super-paramagnetic beads $\left(2 a=1 \mu \mathrm{m}, \rho_{b}=2250 \mathrm{~kg} / \mathrm{m}^{3}, \chi=1.3\right)$ suspended in water $\left(\eta=10^{-3} \mathrm{~kg} / \mathrm{ms}, \rho_{w}=1000 \mathrm{~kg} / \mathrm{m}^{3}\right)$ within a uniform magnetic field $(\mathbf{B}=11 \mathrm{mT})$ in the horizontal direction at room temperature using a time step of $10^{-6} \mathrm{~s}\left(d t=10^{-6} \mathrm{~s}\right)$.

\section{Material and methods}

\subsection{Super-paramagnetic Bead}

Super-paramagnetic beads, (SiMAG - Silanol), $1 \mu \mathrm{m}$ sized, made of a maghemite core surrounded by a silica surface, were purchased from Chemicell (Berlin, Germany) at a concentration of $9 \times 10^{10}$ beads $/ \mathrm{ml}$.

The magnetic response of the beads was measured using a SQUID magnetometer (Quantum Design 7T Magnetic properties Measurement System (MPMS) XL). The magnetic response of the magnetic beads is shown on figure 1.

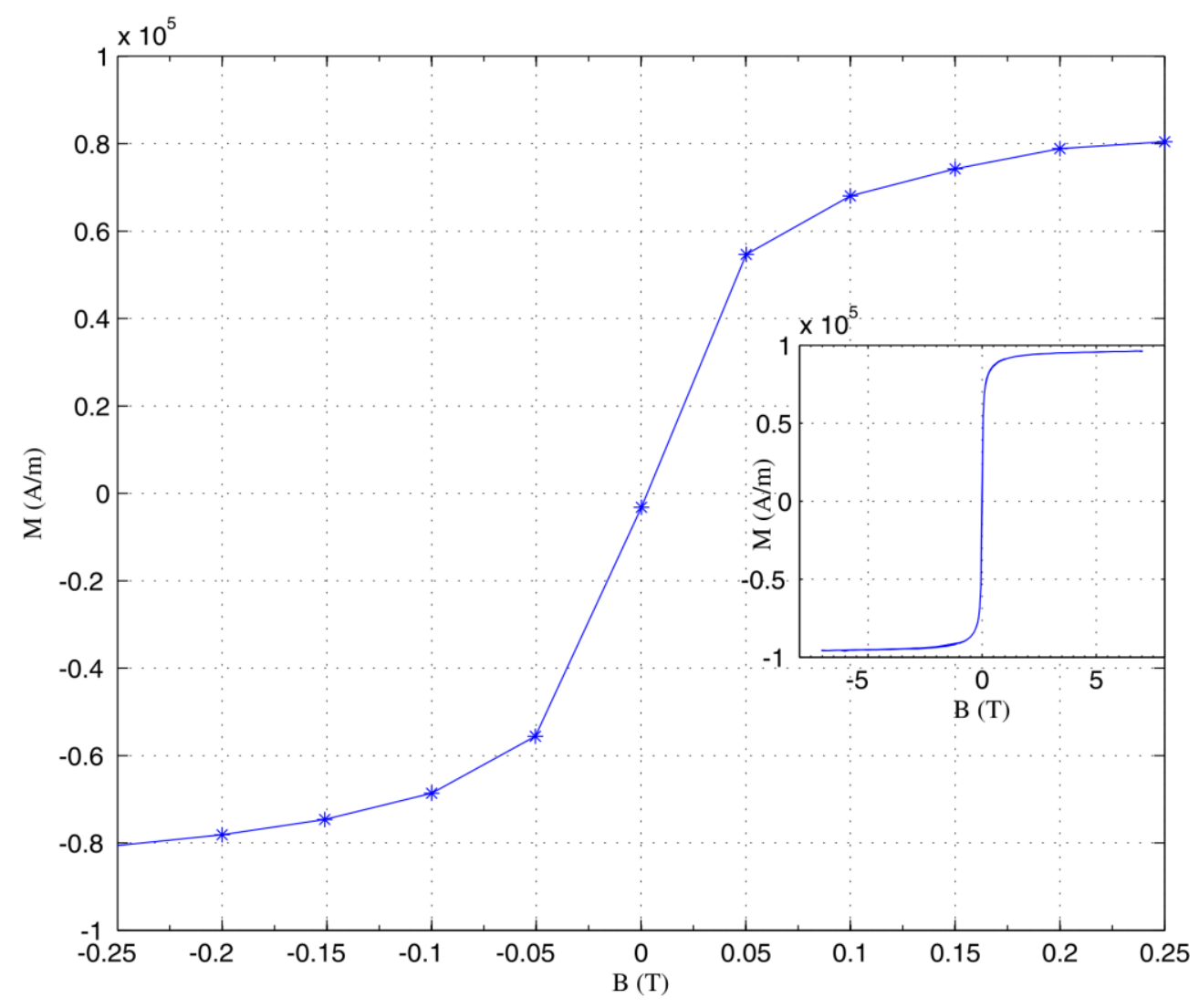

Figure 1. A relationship between the magnetization, $\mathbf{M}$ and the applied flux density, $\mathbf{B}$ of a volume containing a large number of $1 \mu \mathrm{m}$ sized super-paramagnetic bead obtained from Chemicell, at room temperature within the range $\pm 0.25 \mathrm{~T}$. The inset figure shows the magnetization versus applied flux density characterization within the range $\pm 7 \mathrm{~T}$.

A linear interpolation within the applied flux density range $( \pm 50 \mathrm{mT})$ was used to estimate the magnetic susceptibility of the bead $(\chi=1.3)$ at $11 \mathrm{mT}$ due to the limited data points. 


\subsection{Static Fluid Cell}

Counting slides with physical dimensions of $85 \mathrm{~mm}$ by $40 \mathrm{~mm}$ by $0.1 \mathrm{~mm}$ were obtained from Immune Systems (UK). These were cut and separated into individual cells for the experiment.

\subsection{Sample Preparation and Particle Tracking}

A solution of super-paramagnetic beads (Chemicell) in deionized water was freshly prepared and diluted to achieve a concentration of $9 \times 10^{3}$ beads $/ \mathrm{ml}$; this concentration was confirmed by observing the number of beads within the view of the microscope. The bead solution was placed in an ultrasonic bath for 5 minutes to break up all possible aggregates and fully suspend the particles. A volume of $6 \mu \mathrm{l}$ of the solution was transferred to the hydrostatic fluid observation channel using an appropriate pipette.

A uniform magnetic field was achieved by placing two rectangular $(20 \mathrm{~mm}$ by $10 \mathrm{~mm}$ by $5 \mathrm{~mm}$ N42 Neodymium Magnets) with magnetic field strength of $0.58 \mathrm{~T}$ separated by a distance of $55 \mathrm{~mm}$ in a position of attraction. The applied flux density of the uniform magnetic field was measured using a Hall Effect Gauss/Tesla meter (5100 Series Sypris Test \& Measurement F.W.Bell 12/04) and gave a value of $11 \pm 0.5 \mathrm{mT}$. This is consistent with field values obtained by modelling the distribution in Ansys Maxwell; 11mT has been used in the simulation. The observation channel was placed within the uniform magnetic field distribution along the centre line of the magnets.

Interaction between a pair of isolated magnetic beads was observed within a video window of 1024 by 768 pixels $(1$ pixel $=0.073 \mu \mathrm{m})$ such that interaction with other beads can be neglected. The bead motion was recorded using an inverted microscope (Leitz Wetzlar, Germany) with a long working distance 20x objective lens (Comar Optics, Cambridge, UK) which provided sufficient magnification. A USB digital camera (UEye UI - 2230SE, IDS, Germany) was used to record images and videos of the movement of the beads for 5 minutes. The video frames were analysed using particle tracking software (ImageJ [20]). This experiment was repeated for a higher concentration $\left(9 \times 10^{4}\right.$ beads $\left./ \mathrm{ml}\right)$ which was again verified by counting. The trajectories of the beads were tracked on a frame by frame basis and these showed a large amount of Brownian displacement. In order to evaluate the Brownian displacement, the experiment was repeated with a concentration of $1 \times 10^{4}$ beads $/ \mathrm{ml}$ in the absence of the magnets.

\section{Results and Discussion}

\subsection{Brownian Motion}

When the beads were weakly affected by magnetic interactions, Brownian motion was observed. The root mean square displacement of the super-paramagnetic beads was determined from the video data to be $0.27 \mu \mathrm{m}$ while calculations (equation 9) predict a value of $0.23 \mu \mathrm{m}$ within an interval of $30 \mathrm{~ms}$, corresponding to the camera frame rate. This confirms that, when the beads are sufficiently separated, they are subjected to Brownian motion.

\subsection{Bead Pair Interaction}


The trajectory plot of the bead pair was obtained from the simulation and superimposed with the captured video as presented in figure 2. The dots on figure 2 along the trajectory curves represent simulated beads' position at 30ms intervals whereas the crosses show the actual beads' position using particle tracking per frame. The spacing between the dots give an indication of the simulated velocity. More results can be found within the supplementary information section.

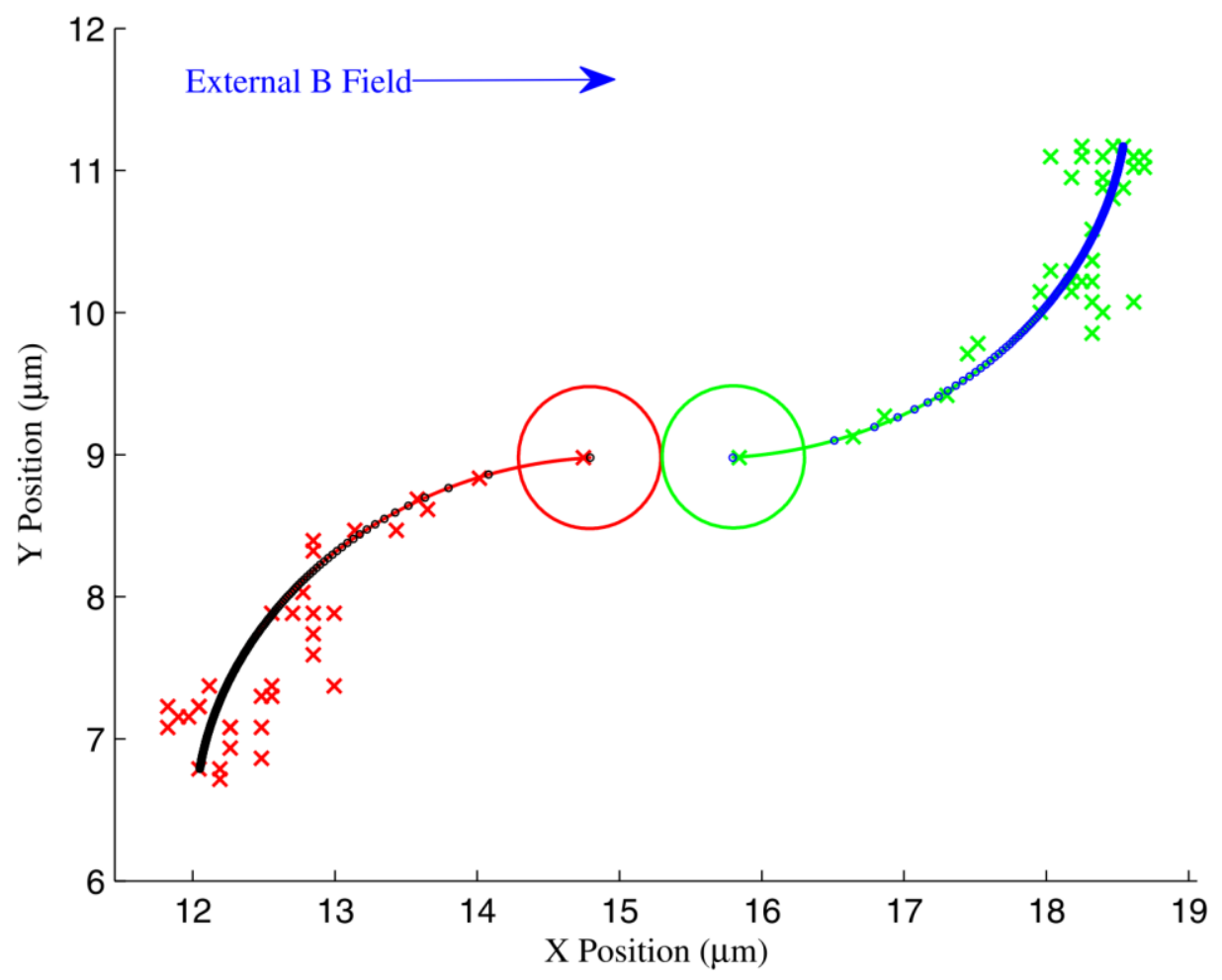

Figure 2. Trajectory of $1 \mu \mathrm{m}$ diameter super-paramagnetic beads moving together under the influence of both magnetic and hydrodynamic interaction within a uniform external magnetic field (direction shown by the blue line) in water; experimental data ( $\times$ crosses) and simulation results (continuous line) are compared to validate the model. The dots (in blue and black) represent a time step of $0.03 \mathrm{~s}$.

It is observed that when the beads are separated by a distance greater than approximately six times their diameter, the interaction is weak and the beads' movement is mainly governed by Brownian motion as indicated by the scattered crosses at the start of the trajectory. The simulation does not attempt to include the Brownian displacement as a statistical variation. As the beads approach one another, their magnetic interaction becomes stronger and they align in the direction of the magnetic field ( $\mathrm{x}$-axis) before approaching to form an agglomerate.

The trajectory plot of the beads shows two regions; a first region where Brownian displacement is noticeable when the beads are further apart and align in the direction of the magnetic field $(>6 \mu \mathrm{m})$ and a second region when this displacement is insignificant due to the increased velocities of the beads as shown on figure 2. This is as a result of the enhanced magnetic force interaction set up between them. Within this region, the beads approach each 
other within a short time interval as indicated by the widely spaced dots in figure 2 . The simulated velocity is consistent with the velocities obtained from the video data at regions where the magnetic interaction dominated the Brownian dynamics. For example, when the beads are momentarily separated by a gap-distance of $2.3 \mu \mathrm{m}$, the simulation predicts a velocity of $12.7 \mu \mathrm{m} / \mathrm{s}$ whereas the average of the velocities of the two beads as determined from the video data at that same instant is $11.75 \mu \mathrm{m} / \mathrm{s}$.

\subsection{Agglomeration Time}

For beads suspended in solution, the time at which they begin to agglomerate depends on the concentration of beads and their initial distribution. Considering two nearest neighbour beads (such as those in this study) positioned along a line in the direction of the magnetic field, simulation predicts the time it takes for the beads to agglomerate as a function of the initial separation distance, as shown on figure 3. This graph can be used to estimate the agglomeration time for a given concentration.

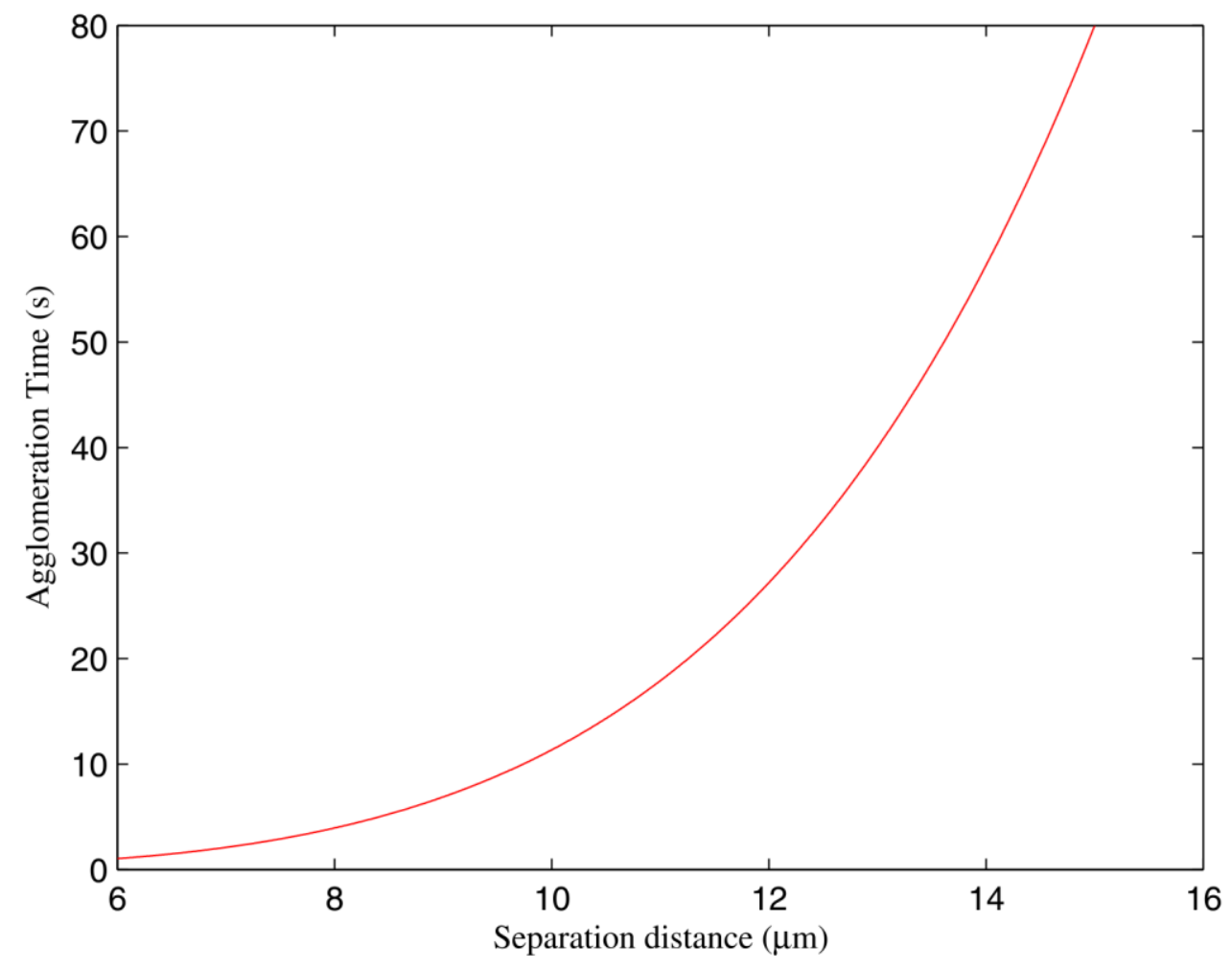

Figure 3. Relationship between the agglomeration time and separation distance for a pair of $1 \mu \mathrm{m}$ Chemicell beads positioned along the direction of the magnetic field.

\section{Conclusion}

A theoretical analysis of the interactions between a pair of magnetic beads in a magnetic separation system has been investigated; this led to the development of a two dimensional 
numerical model in Matlab. A video was captured showing a pair of magnetic beads suspended in a static fluid interacting within a uniform magnetic field.

A good agreement was obtained by comparing the data from the captured video with the simulation. A conclusion can be reached that the model is a good approximation for particle interactions in a magnetophoretic system based on the assumption that the magnetic field is sufficiently small that a linear relationship exists between the magnetization and the external magnetic field.

Furthermore, the model is able to predict the trajectory pattern of magnetic beads suspended within a low concentration for a uniform magnetic field.

We can therefore conclude that agglomeration begins when the attracting magnetic interaction has sufficient time to work against the restraining hydrodynamic force between beads suspended in a fluid. This study shows that the magnetic attraction dominates Brownian motion when the Chemicell beads (SiMAG - Silanol 1101-1) become separated by a gap-distance of six times their diameter. The time taken for a pair of beads to come together when evaluated for different separation distances can be used to estimate the agglomeration time for bead assays of different concentration.

This model is being extended to a three dimensional model with more particles interacting within a magnetic chamber in order to investigate the separation efficiency of a magnetic separation system and avoid the formation of agglomerates.

\section{Acknowledgments}

The corresponding author gratefully acknowledges the support of Emmanuel TV for funding.

\section{References}

[1] N. Pamme, Magnetism and microfluidics, RSC Labchip J.6 (2005) 24-38.

[2] E. P. Furlani, Analysis of particle transport in a magnetophoretic microsystem, J. Appl. Phys. 99 (2006).

[3] D. W. Inglis, R. Riehn, R. H. Austin, J. C. Sturm, Continuous microfluidic immunomagnetic cell separation, Appl. Phys. Lett. 85 (2004) 5093-5095.

[4] Y. Haik, V. Pai, C. Chen, Development of magnetic device for cell separation, J. Magn. Magn. Mater. 194 (1999) 254-261.

[5] K. Han, A. B. Frazier, Continuous magnetophoretic separation of blood cells in microdevice format, J. Appl. Phys. 96 (2004) 5797-5802.

[6] Y. Zhou, Y. Wang, Q. Lin, A Microchip for Continuous-Flow, Magnetically Controlled Capture and Separation of Microparticles, Colombia University, CFD Research Corporation,http://biomems.me.columbia.edu/research/PDFs/jour/ZhouY09_cMACS.pdf, last assessed on 25/08/15. 
[7] K. Nandy, S. Chaudhuri, R. Ganguly, I. K. Puri, Analytical model for the magnetophoretic capture of magnetic microspheres in microfluidic devices, J. Magn. Magn. Mater. 320 (2008) 1398-1405.

[8] N. Pamme, A. Manz, On-Chip Free-Flow Magnetophoresis: Continuous Flow Separation of Magnetic Particles and Agglomerates, Anal. Chem. 76 (2004) 7250 - 7256.

[9] N. Wise, T. Grob, K. Morten, I. Thompson, S. Sheard, Magnetophoretic velocities of superparamagnetic particles, agglomerates and complexes, J. Magn. Magn. Mater. 384 (2015) $328-334$.

[10] C. Mikkelsen, M. F. Hansen, H. Bruus, Theoretical comparison of magnetic and hydrodynamic interactions between magnetically tagged particles in microfluidic systems, J. Magn. Magn. Mater. 293 (2005) 578-583.

[11] U. Banerjee, P. Bit, R. Ganguly, S. Hardt, Aggregation dynamics of particles in a microchannel due to an applied magnetic field, J. Microfluid Nanofluid 13 (2012) 565 577.

[12] S. Nasseri, N. Phan-Thien, Hydrodynamic interaction between two nearby swimming micromachines, J. Comp. Mech. 20 (1997) 551-559.

[13] J. Happel, H. Brenner, Low Reynolds number in hydrodynamics with special applications in particulate media, first paperback ed., Martinus Nijhoff Publishers, Netherlands, 1983.

[14] S. Yang, L. G. Leal, Y. Kim, Hydrodynamic Interaction between Spheres Coated with Deformable Thin Liquid Films, J. Coll. Int. Sci. 250 (2002) 457-465.

[15] L. L. Gary, Advanced Transport Phenomena: fluid mechanics and convective transport processes, first ed., Cambridge University Press, Cambridge, 2007.

[16] A. Sinha, R. Ganguly, I. K. Puri, Magnetic separation from superparamagnetic particle suspensions, J. Magn. Magn. Mater. 321 (2009) 2251-2256.

[17] D. Liu, M. R. Maxey, G. E. Karniadakis, Simulations of dynamic self-assembly of paramagnetic microspheres in confined microgeometries, J. Micromech. Microeng. 15 (2005) $2298-2308$.

[18] R. A. Dragovic, C. Gardiner, A. S. Brooks, D. S. Tanetta, D. J. P. Ferguson, P. Hole, B. Carr, C. W. G. Redman, A. L. Harris, P. J. Dobson, P. Harrison, I. I. Sargent. Sizing and phenotyping of cellular vesicles using Nanoparticle Tracking analysis, Nanomedicine. Nano. Bio. and Med. 7 (2011) 780 -788.

[19] G. R. Lindfield, J. E. T. Penny, Numerical Methods using Matlab, third ed., Elsevier Academic Press, Amsterdam, London, 2012.

[20] T. Ferreira, W. Rasband, Image J User Guide, FIJI 1.46, October 2012.

[21] J. D. Jackson, Classical Electrodynamics, third ed., John Wiley \& Sons, New York, Chichester, 1999. 


\section{APPENDIX A}

Assuming a spherical super-paramagnetic bead lies within a uniform magnetic field, it can be approximated as a dipole [21] with magnetization given by

$$
\mathbf{M}=\chi_{e f f} \mathbf{H}_{\mathbf{e x t}}, \quad \chi_{e f f}=\frac{3 \chi}{\chi+3}
$$

where $\chi$ is the intrinsic magnetic bead susceptibility while $\mathbf{H}_{\mathbf{e x t}}$ is the external magnetic field.

The magnetic field of a dipole at a position vector, $\mathbf{r}$, is then expressed as

$$
\begin{array}{r}
\mathbf{H}(\mathbf{r})=\frac{1}{4 \pi}\left[\frac{3(\mathbf{m} \cdot \mathbf{r}) \mathbf{r}}{r^{5}}-\frac{\mathbf{m}}{r^{3}}\right] \\
\mathbf{m}=\mathbf{M} V
\end{array}
$$

where $\mathbf{m}$ is the magnetic moment and $V$ is the volume of the bead [21].

If a second magnetic bead is placed within the uniform external field, its presence leads to a change in the magnetization and induced field of the first bead. This induced field can be expressed using equation $\mathrm{A} 2$ as given below.

$$
\mathbf{d H}_{\mathbf{2}}(\mathbf{r})=\chi_{e f f} \frac{a^{3}}{r^{3}} \times\left(\frac{3\left(\mathbf{H}_{\mathbf{e x t}}\left(\boldsymbol{r}_{2}\right) \cdot \mathbf{r}\right) \mathbf{r}}{r^{2}}-\mathbf{H}_{\mathbf{e x t}}\left(\boldsymbol{r}_{2}\right)\right)
$$

The magnetic force, $\mathbf{F}_{\mathbf{m} \mathbf{1}}$, on the first super-paramagnetic bead interacting with the second bead can then be written as

$$
\mathbf{F}_{\mathbf{m} \mathbf{1}}=\int \mu_{o}\left(\left(\mathbf{M}_{\mathbf{1}}+\mathbf{d} \mathbf{M}_{\mathbf{1}}\right) \cdot \boldsymbol{\nabla}\right)\left(\mathbf{H}_{\mathrm{ext}}+\mathbf{d H}_{\mathbf{2}}\right) d V
$$

Equation A5 has been simplified in [10]. For this application (uniform field), the significant interaction force is given by

$$
\mathbf{F}_{\mathbf{m} 1}=\varphi_{0} V\left(\left(\mathbf{M}_{\mathbf{1}} \cdot \boldsymbol{\nabla}\right) \mathbf{d H}_{\mathbf{2}}(\mathbf{r})\right)
$$

